This is the final peer-reviewed accepted manuscript of:

[Chiral analysis of theanine and catechin in characterization of green tea by cyclodextrin-modified micellar electrokinetic chromatography and high performance liquid chromatography, Jessica Fiori, Benedetta Pasquini, Claudia Caprini, Serena Orlandini, Sandra Furlanetto, Roberto Gotti, Journal of Chromatography A, 1562 (2018) 115-122]

The final published version is available online at: [doi.org/10.1016/i.chroma.2018.05.063]

Rights / License:

The terms and conditions for the reuse of this version of the manuscript are specified in the publishing policy. For all terms of use and more information see the publisher's website.

This item was downloaded from IRIS Università di Bologna (https://cris.unibo.it/)

When citing, please refer to the published version. 


\title{
Chiral analysis of theanine and catechin in characterization of green tea by cyclodextrin-modified micellar electrokinetic chromatography and high performance liquid chromatography ${ }^{\text {is }}$
}

\author{
Jessica Fiori ${ }^{\mathrm{a}}$, Benedetta Pasquini ${ }^{\mathrm{b}}$, Claudia Caprini ${ }^{\mathrm{b}}$, Serena Orlandini ${ }^{\mathrm{b}}$, \\ Sandra Furlanetto ${ }^{\mathrm{b}}$, Roberto Gotti ${ }^{\mathrm{c}, *}$ \\ a Department of Chemistry “Giacomo Ciamician”, University of Bologna, Via Selmi 2, 40126, Bologna, Italy \\ b Department of Chemistry “U. Schiff”, University of Florence, Via U. Schiff 6, 50019, Sesto Fiorentino, Florence, Italy \\ c Department of Pharmacy and Biotechnology, University of Bologna, Via Belmeloro 6, 40126, Bologna, Italy
}

\section{A R T I C L E I N F O}

\section{Article history:}

Received 30 March 2018

Received in revised form 21 May 2018

Accepted 27 May 2018

Available online $\mathrm{xxx}$

\section{Keywords:}

$( \pm)$-Catechin

$\mathrm{D} / \mathrm{L}$-Theanine

Derivatization

Chiral analysis

Micellar electrokinetic chromatography HPLC

\begin{abstract}
A B S T R A C T
Monomeric catechins are important compounds in green tea accounting for potential bioactivity against a wide range of diseases. Besides catechins, L-Theanine ( $\gamma$-glutamylethylamide), a characteristic amino acid in tea leaves, has become a further focus of the phytochemical research for the reported beneficial effects mainly on cognitive performance, emotional state and sleep quality. In the present study has been developed a CD-MEKC method based on sodium dodecyl sulfate (SDS) and Heptakis (2,6-di-Omethyl)- $\beta$-cyclodextrin for the separation of six major green tea catechins and enantiomers of theanine. The latter, because of the poor detectability was derivatized prior analysis by o-phthaldialdehyde in the presence of $\mathrm{N}$-acetyl-L-cysteine which, under mild conditions (neutral $\mathrm{pH}$, in two minutes) allowed two diastereomers isoindole derivatives to be obtained. The derivatization reaction was directly carried out on tea infusion and derivatized samples were analysed by CD-MEKC involving $65 \mathrm{mM}$ SDS and $28 \mathrm{mM}$ cyclodextrin in acidic buffer ( $\mathrm{pH} 2.5$ ). The separation of six major green tea catechins including enantioresolution of $( \pm)$-Catechin and $\mathrm{D} / \mathrm{L}$-Theanine was obtained in about $5 \mathrm{~min}$ allowing $\mathrm{D}$-Theanine to be quantified at least at $0.5 \% \mathrm{~m} / \mathrm{m}$ level with respect to $\mathrm{L}$-Theanine. Since (-)-Catechin and $\mathrm{D}$-Theanine can be considered as non-native enantiomers (distomers), their presence in real samples provides an indication of tea leaves treatments (thermal treatment, fermentation, etc.) and could represent an opportunity for grading tea. The obtained results were confirmed by a RP-HPLC approach; even though the chromatography was developed in achiral conditions, the derivatization approach applied to theanine (diastereomers formation), allowed for $\mathrm{D} / \mathrm{L}$-Theanine chiral analysis.
\end{abstract}

(C) 2018 Published by Elsevier B.V.

\section{Introduction}

Green tea, the water infusion of unfermented leaves of Camellia sinensis (L.) Kuntze, is the most popular beverage in Asia and its consumption in west countries is continuously increasing. The worldwide acceptance of green tea can be associated to the claimed health promoting effects, mainly attributed to catechins, exerted against a wide range of human diseases such as cancer, cardiovascular diseases and other degenerative disorders $[1,2]$. The major green tea monomeric catechins are: $(+)$-catechin (+)-C, (-)-epicatechin (-)-EC, (-)-epicatechin gallate (-)-ECG, (-)-

\footnotetext{
t5 Thematic Virtual Special Issue on "Enantioseparations-2018".

* Corresponding author.

E-mail address: roberto.gotti@unibo.it (R. Gotti).
}

epigallocatechin (-)-EGC, (-)-epigallocatechin gallate (-)-EGCG, (-)-gallocatechin gallate (-)-GCG, (+)-gallocatechin (+)-GC. Quantification of these bioactive compounds can be performed using RP-HPLC and nanoLC also hyphenated to mass spectrometry [3-8] and by capillary electrophoresis (CE) [9] to evaluate the quality/biological value of the tea products as well as to differentiate the origin and fermentation degree [10-13], to evaluate the influence of seasonal variations [14] and the effects of processing and storage on the polyphenols content [15].

Besides catechins, amino acids are the most important components of green tea and being involved in aroma formation, their quantity in leaves and derived beverages can be considered as a primary factor affecting the quality of the commercial products [9]. L-Theanine ( $\gamma$-glutamylethylamide; L-Th) is the most characteristic amino acid of the genus Camellia beyond which, it has been 
only reported in Xerocomus badius, a kind of nonedible mushroom. Theanine represents more than $50 \%$ of all the amino acids content, and it is responsible for the sweet and brothy - or savory taste (defined as umami) of tea $[16,17]$. The potential health effects of theanine have become a forefront in the study of the beneficial activity associated to tea consumption, since it has proved to exert relaxation by improving cognitive performance, emotional state and sleep quality [16-18]. Establishing the quantity of theanine is a major step in grading tea, indeed in 2017, the International Standardization Organization (ISO) has released a method for its quantitation by means of RP-HPLC [19]. CE using detection at 205 and $214 \mathrm{~nm}$ was applied for the direct quantitation of theanine [20,21], however improved sensitivity was obtained by derivatization of tea samples using different labeling reagents for laser induced fluorescence (LIF) detection [22,23]. Focusing on the importance of theanine content in differentiating among tea varieties, derivatization using OPA and 2-mercaptoethanol (ME) has been applied in HPLC [24-26]. Recently, Horanni and Engelhardt determined the content of theanine in different kinds of tea samples (white, green, black, oolong, pu-erh and tea products) using 9fluorenylmethyloxycarbonyl chloride (FMO-Cl) in HPLC, reporting levels within $0.07-20 \mathrm{mg} / \mathrm{g}$ (dry weight, dw) [27]. The mentioned studies did not focus on the enantioseparation of L-Theanine (LTh) and D-Theanine (D-Th), which however should be considered to provide information on sample stability. Indeed, the native form L-Th, was found to undergo racemization as investigated by EkborgOtt et al., in a number of tea samples such as green, half green and black teas. The separation of theanine enantiomers was achieved by derivatization using FMO-Cl followed by chromatography performed in a system comprising of a reversed phase C18 column, coupled to a $\gamma$-Cyclodextrin column used in polar-organic mode. It was observed that in some types of tea samples, low absolute amounts of the native enantiomer were associated with high percentages of the distomer D-Th. It was argued that during handling and processing of the tea samples, microorganisms present in the leaves, could selectively utilize or degrade L-Th as well as promote a fermentation leading to racemization [28]. More recently, the study on the correlation between tea quality/grade and enantiomeric composition of theanine, has been extended by applying HPLC for separation of the diastereomers obtained by derivatization of theanine enantiomers with a chiral Marfey's reagent [29], by direct enantioresolution using $\beta$-cyclodextrin as a chiral additive to the mobile phase in RP-HPLC [30] and by using a teicoplanin chiral stationary phase for HPLC separation combined to atmospheric pressure ionization mass spectrometry (API-MS) [31]. In our previous studies, cyclodextrin-modified micellar electrokinetic chromatography (CD-MEKC) was applied to the characterization of green tea samples by evaluation of the quantitative profile of catechins. In particular, the combination of the surfactant sodium dodecyl sulfate (SDS) with the chiral selector (2-hydroxypropyl)$\beta$-cyclodextrin (HP $\beta C D)$ in an acidic background electrolyte $(\mathrm{pH}$ 2.5), allowed the resolution of (-)-EC, (-)-ECG, (-)-EGC, (-)-EGCG, $(-)-G C G$, including the enantioseparation of $( \pm)-C$ and $( \pm)-G C$ $[12,13,15]$. Being (-)-C and (-)-GC non-native enantiomers (not represented in plant kingdom) [32,33], their presence was assumed as a proof of thermal treatment and to estimate aging and storage conditions of the commercial samples. However the applied methods were not aimed at the analysis of theanine, which as well, is an appropriate indicator for grading tea. Thus in the present study, a CD-MEKC method was developed focusing on the simultaneous enantioselective analysis of the major amino acid theanine and that of catechin, to investigate more in deep the chiral stability of green tea constituents and to characterize different tea types. In order to improve the detectability of theanine, derivatization using OPA in the presence of N-acetyl-L-cysteine (NAC) was carried out on the real samples (tea infusion) under mild conditions. The optimized
CD-MEKC method was validated and applied to different tea types showing that the content of distomers (-)-C and D-Th were not correlated each other. While the presence of $(-)-C$ can be considered as a product of thermal epimerization of (-)-EC, that of D-Th might be ascribed to racemization processes (fermentation, microbial or enzymatic activities) that do not necessarily involve heating.

\section{Materials and methods}

\subsection{Materials}

The reference compounds i.e., (+)-C, ( \pm )-C, (-)-EC, (-)-EGC, (-)-ECG, (-)-EGCG, (-)-GCG, L-Th and potassium sorbate (the internal standard), were from Sigma-Aldrich (St. Louis, MO, USA). Reagents used for synthesis of the standard compounds and derivatization reaction i.e., 2-pyrrolidone-5-carboxylic acid, (R)-(+)-2-pyrrolidone-5-carboxylic acid, ethylamine 70\% (aqueous solution), OPA and NAC, were from Sigma-Aldrich. D-Th and $\mathrm{D} / \mathrm{L}-\mathrm{Th}$ were synthesized according to previous methods $[28,29]$. The components of electrophoretic and HPLC buffers i.e., boric acid, phosphoric acid, trifluoroacetic acid (TFA), sodium dodecyl sulfate (SDS), methanol, acetonitrile, $\beta$-cyclodextrin $(\beta C D)$, (2-hydroxypropyl)- $\beta$-cyclodextrin (HP $\beta C D)$, Heptakis (2,6-di-Omethyl)- $\beta$-cyclodextrin (DM $\beta C D)$, Heptakis (2,3,6-tri- $O$-methyl)$\beta$-cyclodextrin (TM $\beta C D)$, were purchased from Sigma-Aldrich. Commercial green tea samples were collected locally in specialized stores.

Ultrapure water used for the preparation of running buffers, samples, standard solutions and tea infusions, was purified by Elix Systems (Millipore, Billerica, MA, USA).

\subsection{Solutions, derivatization reaction and preparation of tea samples}

Standard stock solutions of catechins and theanine $(1 \mathrm{mg} / \mathrm{mL})$ were prepared in a mixture of methanol/water in 10:90 ratio $(\mathrm{v} / \mathrm{v})$. All prepared stock solutions were stored at $-20^{\circ} \mathrm{C}$ for one week. Working standard solutions were prepared daily by appropriate dilution with water in order to obtain the desired final concentrations.

Derivatization reagent was prepared daily by dissolving $5.8 \mathrm{mg}$ of NAC and $10 \mathrm{mg}$ of OPA in $1 \mathrm{~mL}$ of a mixture borate buffer $0.4 \mathrm{M}$ $(\mathrm{pH} 7.5) /$ methanol, 90:10 ratio (v/v). Aliquots of $70 \mu \mathrm{L}$ of standard and/or sample solutions were mixed with $10 \mu \mathrm{L}$ of derivatization reagent and kept at room temperature for $2 \mathrm{~min}$ before dilution using $80 \mu \mathrm{L}$ of the internal standard solution constituted of potassium sorbate ( $1 \mathrm{mg} / \mathrm{mL}$ in water). The final obtained clear solutions were directly injected into the CE apparatus for the analysis.

The tea leaves were finely ground by a vibration mill for 2 min and were then subjected to a general brewing method consisting in the extraction of $1.0 \mathrm{~g}$ of the tea powder with about $40 \mathrm{~mL}$ of $85^{\circ} \mathrm{C}$ water for $7 \mathrm{~min}$. The infusion was cooled, transferred in a volumetric flask and the residue leaves were extracted with water. The collected extracts were completed to volume $(50 \mathrm{~mL})$ with water. The infusion was filtered through a syringe filter $(0.45 \mu \mathrm{m}$ regenerated cellulose by Kinesis, Abreg S.r.L., Italy) before derivatization.

\subsection{CE instrumentation and analysis}

The instrument used for CE analysis was a HPCE from Agilent Technologies (Waldbronn, Germany) using the software Rev. A. 09. 01. Agilent Chemstation. Fused-silica capillaries (Composite Metal Service, Ilkley, UK) were $48.5 \mathrm{~cm}$ total length and $8.5 \mathrm{~cm}$ effective length, with the inner diameter of $50 \mu \mathrm{m}$. Voltage and temperature were set at $30 \mathrm{kV}$ and $30^{\circ} \mathrm{C}$, respectively. The CE sampling was performed by the short-end hydrodynamic injection mode at 
50 mbar for $5 \mathrm{~s}$; the detection wavelength was $220 \mathrm{~nm}$. The used BGE was composed of a $25 \mathrm{mM}$ borate-phosphate buffer at $\mathrm{pH} 2.5$, supplemented with $65 \mathrm{mM}$ SDS and $28 \mathrm{mM}$ DM $\beta C D$.

\subsection{HPLC instrumentation and analysis}

HPLC separations were carried out using a Liquid Chromatograph by Agilent $1050 \mathrm{Ti}$ series (Agilent Technologies) equipped with a DAD detector (detection was set at 230 and $280 \mathrm{~nm}$ ). The stationary phase was a core-shell type Kinetex PFP (pentafluorphenyl) $150 \times 4.6 \mathrm{~mm}(5 \mu \mathrm{m}, 100 \AA)$ by Phenomenex (Castelmaggiore, Bologna, Italy). Analyses were performed under gradient elution using a mobile phase composed of acetonitrile $(A)$ and aqueous TFA $0.1 \% \mathrm{v} / \mathrm{v}(\mathrm{B})$ : from $10 / 90(\mathrm{~A} / \mathrm{B})$ to $20 / 80(\mathrm{~A} / \mathrm{B})$ in $10 \mathrm{~min}$ at the flow rate of $1 \mathrm{~mL} / \mathrm{min}$. Sample injections were manually done by a Rehodyne Model 7125 injector (volume $20 \mu \mathrm{L}$ ).

\section{Results and discussion}

The aim of the present study was the chiral characterization of green tea by the simultaneous analysis of the major catechins including enantioresolution of $( \pm)-C$ and that of the main amino acid D/L-Th. Because of the different nature and electrophoretic behavior of the considered analytes, together with the necessity to provide the separation system with the suitable enantioselectivity, CD-MEKC using SDS as the surfactant in the presence of neutral cyclodextrins as chiral additives, was selected [34,35].

\subsection{Derivatization of theanine using OPA and NAC}

The estimation of enantiomeric composition of $\mathrm{D} / \mathrm{L}-\mathrm{Th}$, requires the detection of the distomer (D-Th) at very low level (e.g., $0.5 \%$, with respect to the eutomers L-Th), thus high sensitivity is necessary and to this purpose, a derivatization approach was carried out. The conventional reaction conditions reported for amino acids labelling in tea samples were not suitable for the simultaneous catechins analysis, being the latter rapidly oxidized to corresponded by-products in the alkaline media necessary for derivatization. Indeed, Wang et al., reported that the HPLC analysis of amino acids in tea samples using OPA at pH 9.5 as a reagent, was strongly affected by the presence of polyphenols producing several unidentified chromatographic peaks hindering the selective analysis of the target molecules [25]. However, since OPA is reported to react with amino acids in a wide range of conditions, we evaluated the optimization of the derivatization procedure at lower $\mathrm{pH}$ values in order to preserve catechins from oxidation/degradation both in labelling step and during the subsequent sample storage. In addition, instead of using ME as the conventional OPA co-reagent, the chiral thiol NAC was employed, enabling diastereomeric isoindole $\mathrm{D} / \mathrm{L}-\mathrm{Th}$ derivatives to be formed thus making more feasible their resolution (see Scheme I in Supplementary Material) [36,37].

In the present investigation, the time course of the derivatization of theanine using OPA and NAC was confirmed to be very fast (less than $2 \mathrm{~min}$ ). The reaction conditions optimization was carried out by considering that the average/maximum content of theanine in real samples is reported to be $1-3 \%$ on the dry leaves [16-18,20-27]. The investigation on the reactivity of the mixture OPA/NAC (in borate buffer $0.4 \mathrm{M}$ ) with L-Th was thus carried out in standard solutions of the amino acid (about $1 \mathrm{mM}$ ) using a large molar excess of the reagent (OPA/NAC/L-Th of 40/20/1). The pH of the reagent solution affects the protonation status of the amino group of the analyte and as a consequence, the reactivity toward OPA. The evolution of the response (peak area) of L-Th was followed at different $\mathrm{pH}$ values in the range 7.0-9.5, finding that the minimum value allowing for the maximization of the analyte response was $\mathrm{pH}$ 7.3. Further investigations on the optimization of derivatization conditions were carried out using OPA/NAC in borate buffer $0.4 \mathrm{M}$ at $\mathrm{pH} 7.5$, a little bit higher than the border $\mathrm{pH}$ condition. The influence of the molar ratio (OPA/NAC with respect to theanine) on the reaction course was evaluated at the ratios 40/20/1, 20/10/1, $10 / 5 / 1$ and $5 / 2.5 / 1$, showing no significant response variations. Since together $\mathrm{D} / \mathrm{L}-\mathrm{Th}$, other amino acids are contained in the real samples, it was selected to carry out the derivatization using the largest molar ratio OPA/NAC in order to provide the suitable reagent excess for reaction completion of all the amino acids content. The stability of the obtained theanine isoindole derivatives was evaluated by maintaining the derivatized samples at room temperature and no $\mathrm{pH}$ adjustment of reaction mixture was applied; within four hours, no trend for response loss was observed, showing peak area fluctuations within RSD of $3 \%(n=6)$.

\subsection{CD-MEKC conditions}

An acidic BGE (phosphate-borate at pH 2.5) was chosen to avoid catechins oxidation during the electrophoretic run; in these conditions, the dissociation suppression of phenolic groups makes these solutes in neutral form thus requiring for separation, the use of SDS micellar solution as a ionic pseudostationary phase. The applied acidic conditions strongly reduced the electro-osmotic flow and neutral catechins migration occurred according to their partition into the anionic micelles, toward the anodic end of the capillary. In order to achieve adequate analysis time, a very short capillary was used. In the applied constrained system, the addition of cyclodextrins (CDs) was found to be useful for selectivity tuning, owing to their ability for inclusion/complexation of the analytes occurring in competition with the distribution versus the SDS micelles. Further, the chiral nature of CDs empowered the BGE with potential enantioselectivity [34,35]. In order to find the best conditions for simultaneous analysis of green tea catechins (including $( \pm)-C$ ), and resolution of derivatized $\mathrm{D} / \mathrm{L}-\mathrm{Th}$, neutral $\mathrm{CD}$ s i.e., $\beta C D, H P \beta C D$, $T M \beta C D$ and $D M \beta C D$, were considered. CD-MEKC experiments carried out in a $\mathrm{pH} 2.5$ phosphate-borate buffer $(25 \mathrm{mM})$ containing SDS, supplemented with the different neutral CDs, showed that $\beta C D$ and $H P \beta C D$ exhibited enantioselectivity for $( \pm)-C$ but were not able to resolve derivatized $\mathrm{D} / \mathrm{L}-\mathrm{Th}$. On the contrary, TM $\beta C D$

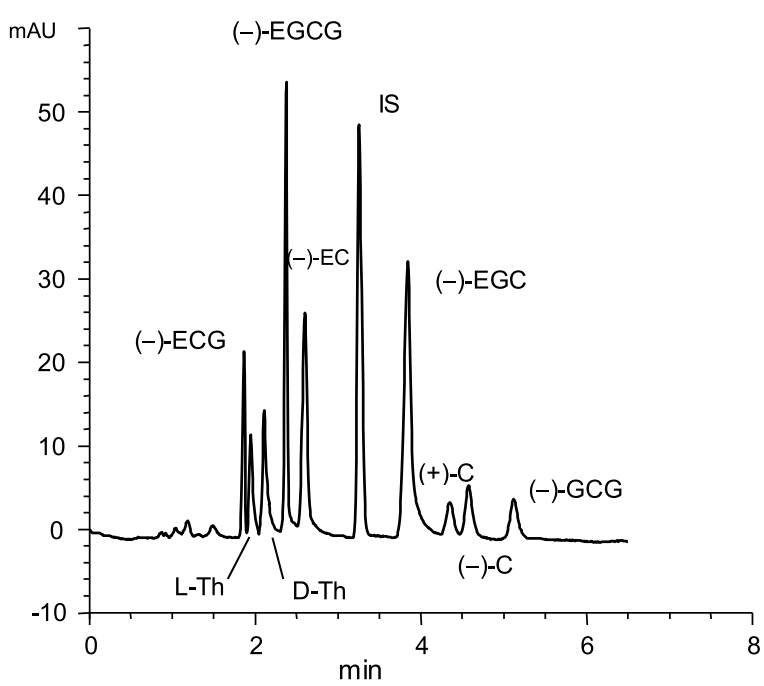

Fig. 1. Electropherogram of standard mixture of catechins and $D / L-T h e a n i n e ~ u p o n$ derivatization using OPA/NAC under optimized CD-MEKC conditions: BGE composed of $\mathrm{pH} 2.5$ phosphate-borate buffer $(25 \mathrm{mM})$ containing $65 \mathrm{mM}$ SDS and $28 \mathrm{mM}$ DM $\beta C D$. Fused-silica capillary $(48.5 \mathrm{~cm}$ total length; $8.5 \mathrm{~cm}$ effective length; i.d. $50 \mu \mathrm{m}$ ); voltage $30 \mathrm{kV}$; capillary cartridge temperature $30^{\circ} \mathrm{C}$; hydrodynamic injection at $50 \mathrm{mbar} \times 5 \mathrm{~s}$; detection at $220 \mathrm{~nm}$. Symbols as in the text. 


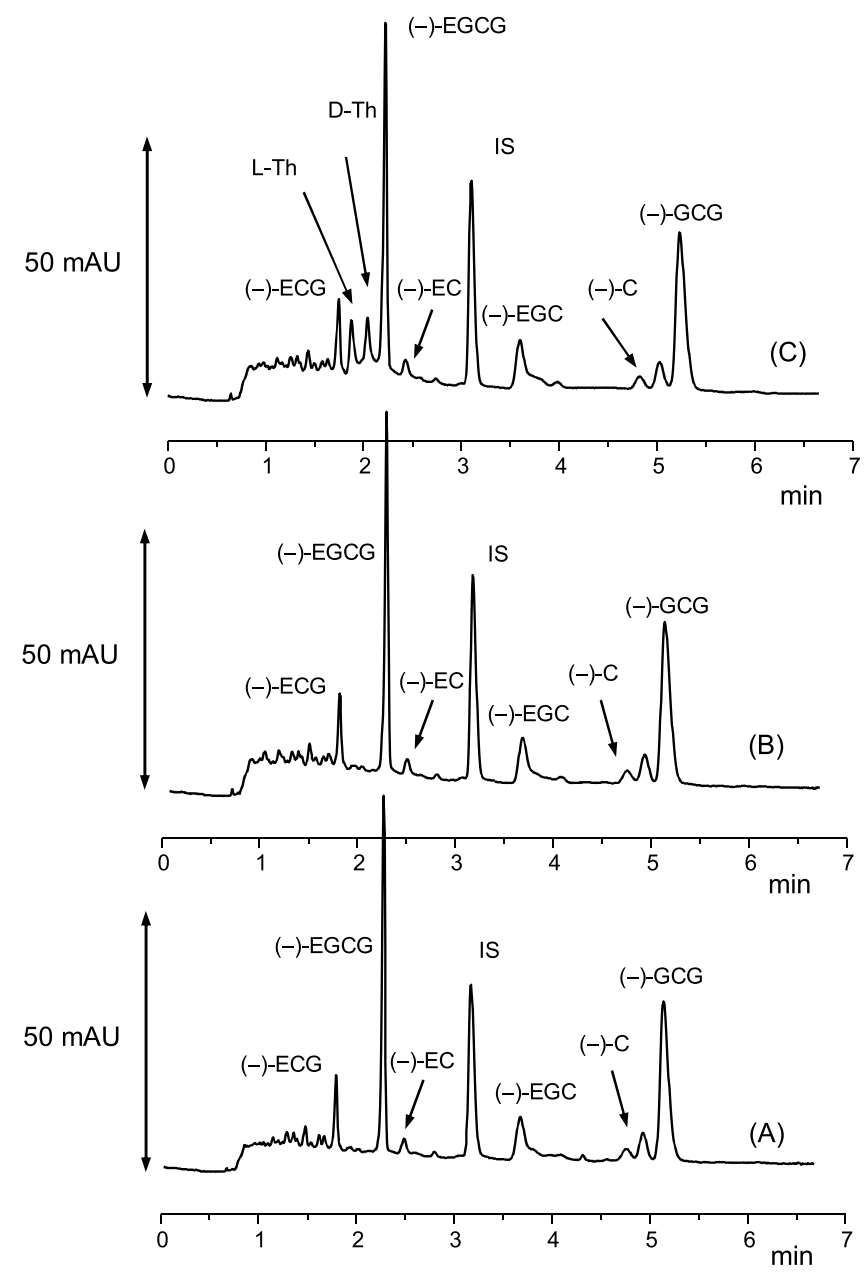

Fig. 2. Electropherograms of Bancha Hojicha tea sample by CD-MEKC; A) analysis before derivatization; B) analysis after derivatization using OPA/NAC; C) analysis of the sample spiked with D/L-Th and derivatized using OPA/NAC. Conditions and symbols as in Fig. 1.

only allowed the enantioresolution of the latter, whereas $( \pm)$-C did not migrate within 20 min. Favorably, in the presence of DM $\beta C D$ the simultaneous enantioresolution of both $( \pm)-C$ and derivatized $\mathrm{D} / \mathrm{L}-\mathrm{Th}$, was achieved. The effect of the composition of the BGE on the separation of a standard mixture of (-)-EC, (-)-EGC, (-)-ECG,
(-)-EGCG, ( \pm )-C, (-)-GCG and derivatized D/L-Th was investigated in the following ranges: phosphate-borate buffer concentration (12.5-50 mM); SDS concentration (50-80 mM); DM- $\beta C D$ concentration (20-40 mM). It was found that the best conditions were: pH 2.5 phosphate-borate buffer $(25 \mathrm{mM})$ containing $65 \mathrm{mM}$ SDS and $28 \mathrm{mM} \mathrm{DM} \beta C D$. Other instrumental parameters as voltage and capillary cartridge temperature were optimized at $30 \mathrm{kV}$ and $30^{\circ} \mathrm{C}$, respectively. The electropherogram of Fig. 1 shows the separation of the standard mixture of the analytes in the presence of potassium sorbate solution as the internal standard. In Fig. 2 it is reported the electropherogram of a green tea sample (Bancha Hojicha tea type) before (Fig. 2A) and after derivatization using OPA/NAC (Fig. 2B); as it can be seen the sample was found to do not contain D/L-Th at the detection limit of the present method. In Fig. 2C it is reported the electropherogram of the same sample spiked with $\mathrm{D} / \mathrm{L}-\mathrm{Th}$ and subjected to derivatization. By the performed experiments it can be concluded that: (i) analysis of D/L-Th is selective and the determination is not affected by matrix components; (ii) the derivatization procedure employing mild conditions ( $\mathrm{pH} 7.5$, room temperature) does not affect the catechins profile.

\section{3. $C D-M E K C$ method validation}

Method validation was performed by estimating linearity, sensitivity, system precision and repeatability, accuracy and robustness, according to ICH (International Council for Harmonization) guidelines [38].

\subsubsection{Linearity and sensitivity}

The linearity ranges of catechins, L-Th and D-Th were selected according to the amount of the compounds in real samples (different green tea types); the equations of the corresponding calibration curves were obtained by plotting the peak area ratio of the analytes to the internal standard, versus their concentration $(\mu \mathrm{g} / \mathrm{mL})$. The data are reported in Table 1: as it can be seen, $\mathrm{r}^{2}$ values obtained by linear regression analysis were at least 0.998 and no trends for residual distribution were observed. Limit of detection (LOD) and limit of quantitation (LOQ) are reported in detail in Table 1 ; the values were estimated by the signal-to-noise ratio defined as 3 (LOD) and 10 times (LOQ) the noise, assumed as the distribution of the response at zero analyte concentration. Regarding catechins, the sensitivity of the method is comparable with that obtained by similar approaches [3,4,7,12,15,33]; higher sensitivity was reported using chromatographic methods hyphenated with MS/MS detection, achieving LOD values in the order of tenths of $\mathrm{ng} / \mathrm{mL}$ [5]. In

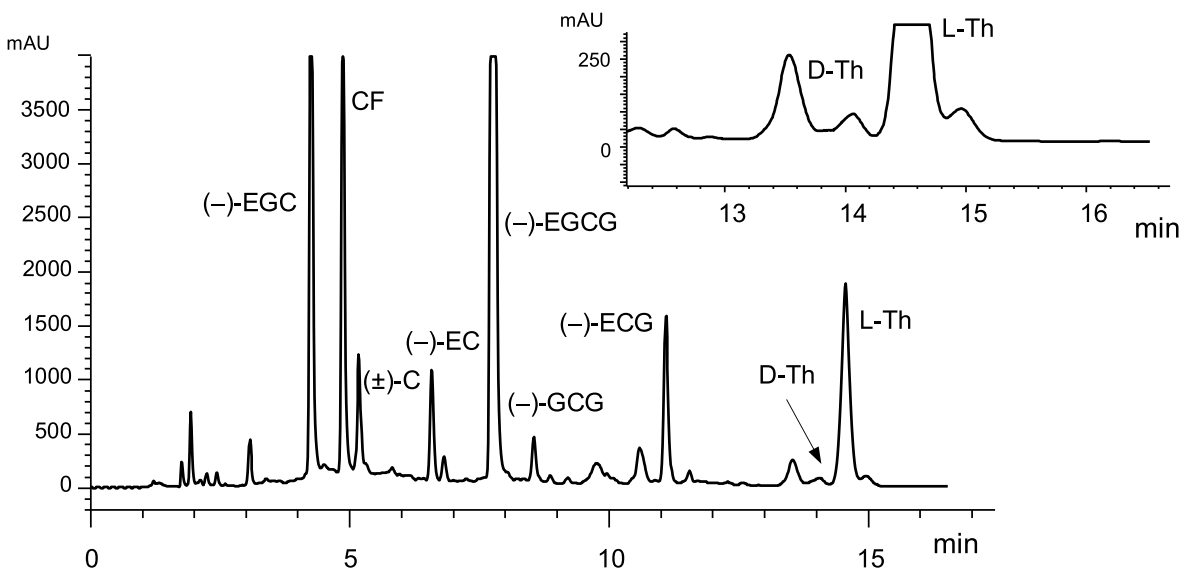

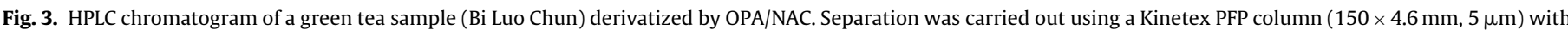

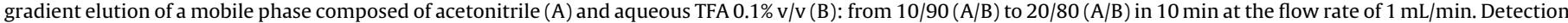

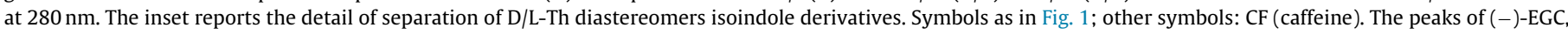
CF and (-)-EGCG are out of the response scale. 
Table 1

Linearity, sensitivity and system precision $(n=9)$ of CD-MEKC method.

\begin{tabular}{|c|c|c|c|c|c|c|}
\hline Analyte & range $\mu \mathrm{g} / \mathrm{mL}$ & Equation & $\mathrm{r}^{2}$ & $\mathrm{LOQ}(\mathrm{RSD} \%) \mu \mathrm{g} / \mathrm{mL}$ & $\mathrm{LOD} \mu \mathrm{g} / \mathrm{mL}$ & $\mathrm{t}_{\mathrm{m}}, \min (\mathrm{RSD} \%)$ \\
\hline$(-)$-ECG & $5.0-150$ & ${ }^{\mathrm{a}} y=0.00754 x+0.00985$ & 0.999 & $0.7(3.5)$ & 0.2 & $1.7(2.08)$ \\
\hline L-Th & $20-400$ & $y=0.00612 x+0.01317$ & 0.999 & $0.7(4.8)$ & 0.2 & $1.9(1.51)$ \\
\hline D-Th & $20-400$ & $y=0.00645 x+0.06212$ & 0.998 & $0.7(5.2)$ & 0.2 & $2.1(1.80)$ \\
\hline$(-)$-EGCG & $40-600$ & $y=0.01084 x+0.1087$ & 0.999 & $0.7(3.4)$ & 0.2 & $2.3(2.27)$ \\
\hline$(-)-E C$ & $10-200$ & $y=0.008470 x+0.0319$ & 0.999 & $0.7(5.0)$ & 0.2 & $2.6(1.27)$ \\
\hline$(-)$-EGC & $15-300$ & $y=0.01307 x+0.03404$ & 0.998 & $0.5(5.4)$ & 0.1 & $3.8(1.25)$ \\
\hline$(+)-C$ & $0.8-24$ & $y=0.00815 x-0.01854$ & 0.999 & $0.6(4.0)$ & 0.2 & $4.3(1.80)$ \\
\hline$(-)-C$ & $0.8-24$ & $y=0.01233 x+0.03504$ & 0.998 & $0.4(4.3)$ & 0.1 & $4.6(1.83)$ \\
\hline$(-)-G C G$ & $2.0-100$ & $y=0.01160 x+0.04041$ & 0.998 & $0.7(5.5)$ & 0.2 & $5.2(4.60)$ \\
\hline
\end{tabular}

a The response $y$, is the peak area ratio of the analytes versus internal standard.

analysis of theanine, the present method showed higher sensitivity with respect to those reported in published CE approaches employing direct UV detection [20,21], whereas lower LOD value (about $0.1 \mathrm{ng} / \mathrm{mL}$ ) was obtained by CE-LIF upon derivatization using 4-chloro-7-nitrobenzofurazan [22]. In general, the HPLC methods showed higher sensitivity with respect to CE analysis reporting LOD of about $10 \mathrm{ng} / \mathrm{mL}$ using both UV and fluorescence detection upon derivatization with OPA/ME [24] and phenylisothiocyanate [26].

To demonstrate the ability of the CD-MEKC method in quantifying D-Th at very low level in the presence of the eutomer L-Th, a standard solution of the latter (about $100 \mu \mathrm{g} / \mathrm{mL}$ ) was spiked with D-Th in the range of $0.5-10 \mu \mathrm{g} / \mathrm{mL}$, showing the opportunity to perform quantification of the distomer at least at $0.5 \%(\mathrm{~m} / \mathrm{m})$ level (see Fig. II Supplementary Material). Among the methods addressed to D/L-Th enantioresolution, only application of LC-ESI/MS of underivatized theanine reported higher detection sensitivity $(10 \mathrm{ng} / \mathrm{mL})$ [31].

\subsubsection{System precision and repeatability}

System precision was assessed by repeated injections $(n=6)$ of a standard mixture of the analytes (concentration at LOQ values). The RSD\% values of migration time as well as those of the estimated LOQs, are reported in Table 1 showing that the instrumental variability at low concentration of the analytes is adequate and consistent with those obtained by CE methods applied to similar samples. The method repeatability estimated by the contribution of sample preparation procedure and analysis, on the whole precision method was assessed by quantifying the considered analytes in three independent samples of a representative green tea (Sencha Ariake type) during three consecutive days. The intra-day $(\mathrm{n}=3)$ and inter-day $(\mathrm{n}=9)$ RSD values were found to range within $0.66-4.10 \%$ and $2.58-7.95 \%$, respectively (Table I Supplementary Material).

\subsubsection{Accuracy}

Accuracy was estimated by recovery experiments performed on Sencha Ariake green tea sample. In details, the considered catechins, L-Th and D-Th, were spiked to the real sample at three levels corresponding to about $50 \%, 100 \%$ and $150 \%$ of their native content, before extraction (brewing) and derivatization. The results are reported in Table II Supplementary Material; the recovery ranged within $86.0-106.8 \%$. The validation data related to precision and accuracy of the present method were in line with those reported using similar methods in analysis of tea samples $[7,12,15]$.

\subsubsection{Robustness}

The electrophoretic method was tested for robustness by evaluating its capability to remain unaffected by small variations of the optimized parameters. In particular, $\mathrm{pH}$ of phosphate-borate buffer used as the BGE and the concentration of SDS and DM $\beta C D$ were the factors to be considered because of their prevalent influence on the separation as observed during optimization experiments. The robustness study was carried out by following a "one-by-one" approach, in which the individual parameters were changed over an established range around the optimized values: phosphateborate buffer $(25 \mathrm{mM}) \mathrm{pH}$ was varied within $2.5 \pm 0.3$ units; SDS and $\mathrm{DM} \beta C \mathrm{C}$ concentrations were varied within $65 \pm 2 \mathrm{mM}$ and $28 \pm 2 \mathrm{mM}$, respectively. The enantioresolutions of $\mathrm{D} / \mathrm{L}-\mathrm{Th}$ and $( \pm)-$ $C$ as well as those of the other critical peaks pairs i.e., (-)-ECG/L-Th; $(-)$-EGCG/(-)-EC and (-)-EGC/(+)-C were maintained at levels allowing for the selective area integration. More in general the method robustness was found to be good, maintaining the resolution between the other couples of adjacent peaks higher than 2.0, in each of the tested conditions.

\subsection{HPLC method}

In order to confirm the results of CD-MEKC approach for chiral analysis of $\mathrm{D} / \mathrm{L}-\mathrm{Th}$, a reversed-phase chromatographic method was developed, actually the two diastereomeric isoindole derivatives obtained by derivatization of $\mathrm{D} / \mathrm{L}-\mathrm{Th}$ can be resolved by achiral stationary phases. Among the conventional tested columns, a core-shell type PFP showed the best performance allowing the complete separation of catechins, caffeine and baseline separation of derivatized D/L-Th in about 15 min by simply applying a linear gradient using acetonitrile/aqueous TFA $0.1 \%$. In Fig. 3 it is shown the chromatogram of a green tea from China (Bi Luo Chun type) derivatized by OPA/NAC according to the proposed method: as it can be seen, the considered sample was found to contain D-Th. The HPLC method was validated for linearity, sensitivity and sys-

Table 2

Content in $\mathrm{mg} / \mathrm{g}(\mathrm{n}=3)$ of catechins and $\mathrm{D} / \mathrm{L}-$ Theanine determined in different tea types by CD-MEKC.

\begin{tabular}{|c|c|c|c|c|c|}
\hline Analyte & Sencha Ariake & Bi Luo Chun & Bancha Hojicha & Oolong & Oolong Formosa \\
\hline$(-)-E C G$ & $4.87 \pm 0.12$ & $4.61 \pm 0.18$ & $1.52 \pm 0.045$ & $3.45 \pm 0.085$ & $1.00 \pm 0.048$ \\
\hline L-Th & $6.55 \pm 0.17$ & $14.85 \pm 0.31$ & - & $0.65 \pm 0.046$ & $8.36 \pm 0.24$ \\
\hline D-Th & - & $2.75 \pm 0.16$ & - & $1.27 \pm 0.068$ & $0.87 \pm 0.036$ \\
\hline (-)-EGCG & $24.21 \pm 0.32$ & $41.88 \pm 1.1$ & $7.61 \pm 0.16$ & $23.48 \pm 0.41$ & $3.83 \pm 0.14$ \\
\hline$(-)-E C$ & $4.01 \pm 0.049$ & $4.29 \pm 0.10$ & $0.78 \pm 0.039$ & $2.76 \pm 0.083$ & $0.982 \pm 0.042$ \\
\hline$(-)$-EGC & $10.91 \pm 0.072$ & $11.54 \pm 0.11$ & $1.52 \pm 0.038$ & $8.51 \pm 0.13$ & $0.988 \pm 0.062$ \\
\hline$(+)-C$ & $0.56 \pm 0.023$ & $1.00 \pm 0.045$ & - & $0.37 \pm 0.018$ & $0.232 \pm 0.019$ \\
\hline$(-)-C$ & - & - & $0.87 \pm 0.074$ & $0.32 \pm 0.022$ & - \\
\hline$(-)-G C G$ & $0.41 \pm 0.01$ & $0.87 \pm 0.026$ & $3.60 \pm 0.11$ & $1.11 \pm 0.035$ & $0.310 \pm 0.015$ \\
\hline
\end{tabular}

Please cite this article in press as: J. Fiori, et al., Chiral analysis of theanine and catechin in characterization of green tea by cyclodextrin-modified micellar electrokinetic chromatography and high performance liquid chromatography, J. Chromatogr. A (2018), https://doi.org/10.1016/j.chroma.2018.05.063 


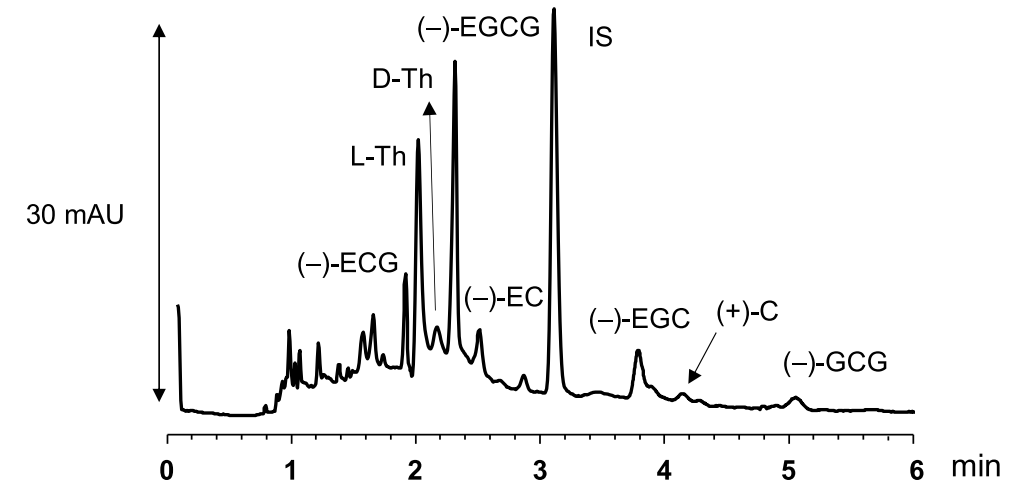

(A)

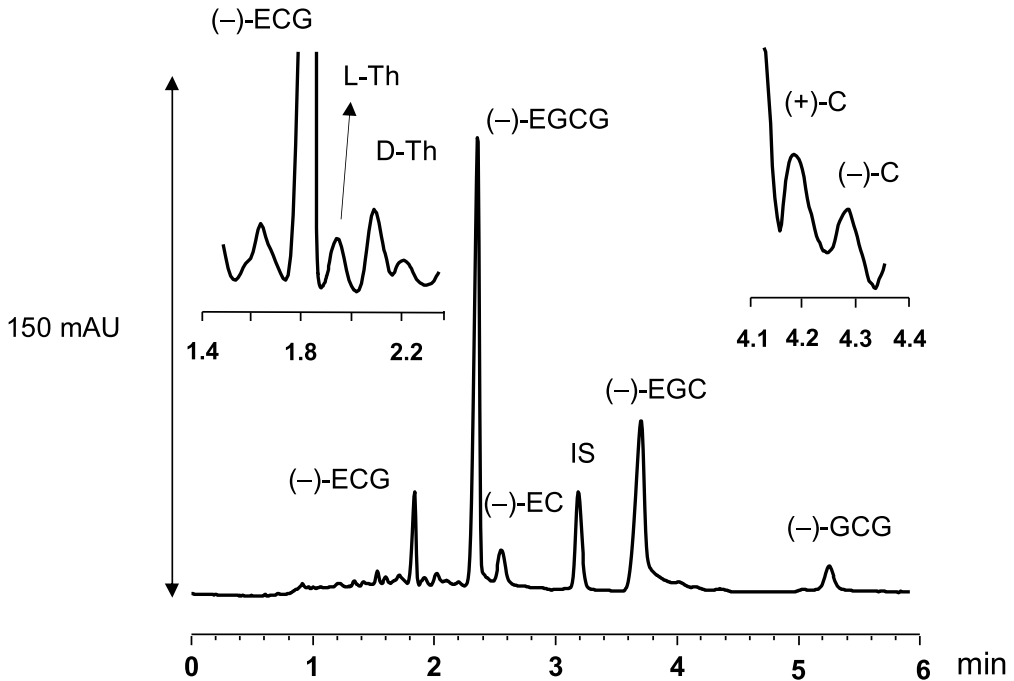

(B)

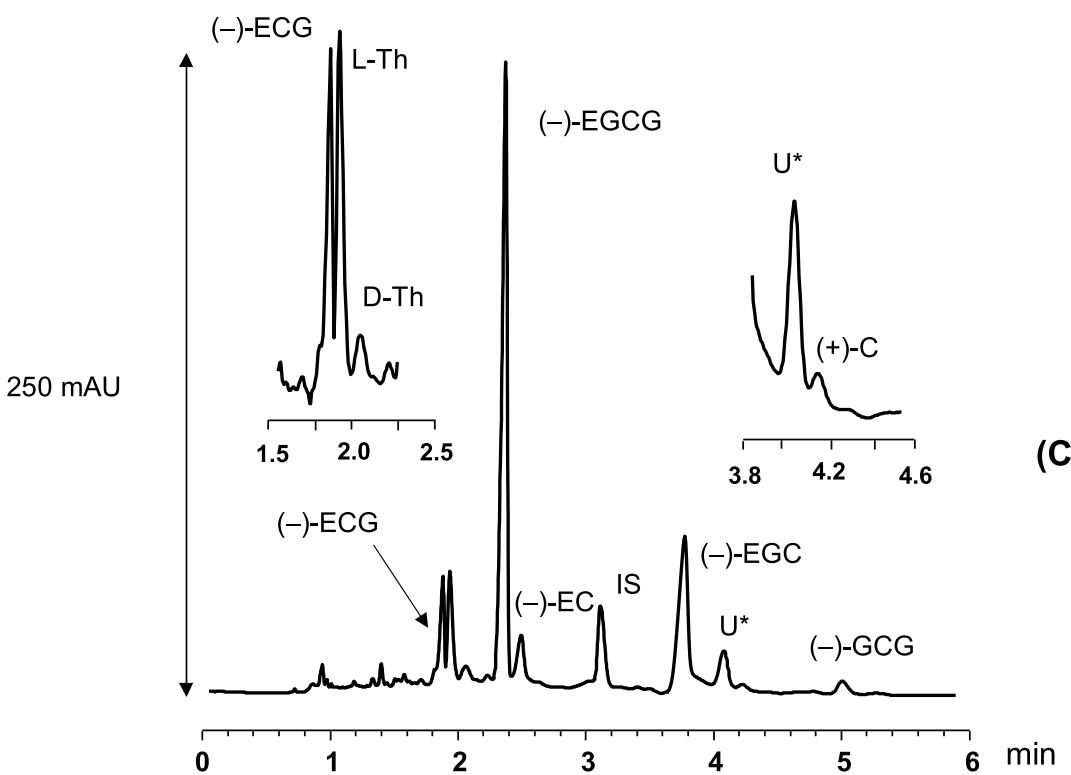

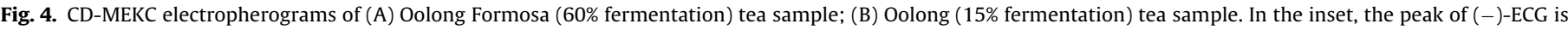
out of the response scale; (C) Bi Luo Chun (green tea as such) sample. $U^{*}=$ unknown; conditions and symbols as in Fig. 1.

tem precision showing the analytical figures of merit reported in detail in Table III Supplementary Material. Owing to the favorable detection flow-cell geometry higher sensitivity was achieved com- pared to CD-MEKC method. The obtained LOD value for D-Th was $0.05 \mu \mathrm{g} / \mathrm{mL}$ at $230 \mathrm{~nm}$, allowing the quantification of the distomer at least at the level of $0.1 \% \mathrm{~m} / \mathrm{m}$ with respect to $\mathrm{L}-\mathrm{Th}$. 


\subsection{Sample analysis}

Thanks to the fast separation time of CD-MEKC method, several green tea types were analysed; according to previous studies, most of them did not show the presence of the distomer D-Th $[28,29]$ and the content of L-Th was found in the range of about $5-18 \mathrm{mg} / \mathrm{g}$ [16-18,20-27]. In the present section, are discussed some results selected to demonstrate the usefulness of the CD-MEKC method in the simultaneous determination of D/L-Th, $( \pm)-C$ and catechins.

In Table 2 are reported the contents of catechins and theanine of five representative tea samples. In green tea as such, the endogenous enzymes (oxidases) in the leaves are inactivated usually by steam immediately after harvesting, in order to prevent fermentation (catechins oxidation) [2,9]; the green tea Sencha Ariake, was thus considered as a typical reference sample. In comparison, Bancha Hojicha tea (Fig. 2B), showed a lower general catechins content, the absence of theanine (both $\mathrm{D}$ and L-enantiomer) and a relatively high level of the distomer (-)-C. The presence of the latter suggested that thermal epimerization of (-)-EC occurred [32,33]; accordingly, the very high level of (-)-GCG with respect to those found in the other tea types, could be ascribed to epimerization of (-)-EGCG. These results are in line with the Bancha Hojicha features, being it defined as "roasted green tea" and subjected to over high heat to improve aroma.

The electropherogram of Fig. 4A, is related to a Oolong Formosa tea, a product that is reported to undergo partial oxidation since inactivation of endogenous enzymes by steam is not immediately performed after harvesting [2]. In particular, a $60 \%$ fermentation degree is declared for the considered sample. As it can be seen by the data of Table 2, most of catechins levels are comparable with those of Bancha Hojicha, however the absence (or trace level) of the distomer (-)-C as well as the amount of (-)-GCG and that of $\mathrm{L}$-Th, were in line with the typical profile of green tea type Sencha Ariake, confirming that the sample was not subjected to high heat/warming manufacturer processes or thermal damaging during storage. Interestingly, the distomer D-Th was found at about $10 \%, \mathrm{~m} / \mathrm{m}$, with respect to L-Th. From one hand, it could be hypothesized that fermentation affects L-Th chiral stability, however this is not confirmed by the results of analysis of a different Oolong tea sample (Fig. 4B) whose fermentation degree was declared to be $15 \%$. In this case as expected, the general catechins content was higher than that of Oolong Formosa (60\% fermentation), conversely the native L-Th was almost completely lost. Finally, a green tea type from China (Bi Luo Chun) showed a catechins content similar and even higher than the reference (Sencha Ariake), including the lack of the distomer (-)-C (Fig. 4C). Interestingly, a relatively high content of D-Th (about $18 \%, \mathrm{~m} / \mathrm{m}$ with respect to L-Th) was found. Even though the reported results are limited to a very restricted number of samples, it can be hypothesized that the presence of $(-)-\mathrm{C}$ is a useful marker of thermal degradation, whereas $D$-Th could rather be the result of microbial or enzymatic processes [28] likely different from oxidases activity, and not necessarily related to thermal treatment.

\section{Conclusion}

CD-MEKC showed to be suitable for the simultaneous analysis of catechins and theanine allowing their enantioseparation in about $5 \mathrm{~min}$. The method was fully validated and the achieved detection limit was sufficient to perform chiral analysis of non-native (-)Catechin and D-Theanine. Conclusive information on the potential biological effects on human health of these distomers are not available from literature, however the results of the present study even though addressed to a limited number of samples, suggest that their integrated/simultaneous determination can be useful in tea characterization.

\section{Acknowledgment}

This work was supported by 2016 (RFO) Ricerca Fondamentale Orientata - University of Bologna, Italy.

\section{Appendix A. Supplementary data}

Supplementary data associated with this article can be found, in the online version, at https://doi.org/10.1016/j.chroma.2018.05. 063.

\section{References}

[1] J.V. Higdon, B. Frei, Tea catechins and polyphenols: health effects, metabolism, and antioxidant functions, Crit. Rev. Food Sci. Nutr. 43 (2003) 89-143.

[2] M. da Silva Pinto, Tea: a new perspective on health benefits, Food Res. Int. 53 (2013) 558-567.

[3] J. Valls, S. Millán, M.P. Martí, E. Borràs, L. Arola, Advanced separation methods of food anthocyanins, isoflavones and flavanols, J. Chromatogr. A 1216 (2009) $7143-7172$.

[4] M. Pelillo, M. Bonoli, B. Biguzzi, A. Bendini, T. Gallina Toschi, G. Lercker, An investigation in the use of HPLC with UV and MS-electrospray detection for the quantification of tea catechins, Food Chem. 87 (2004) 465-470.

[5] W. Tao, Z. Zhou, B. Zhao, T. Wei, Simultaneous determination of eight catechins and four theaflavins in green, black and oolong tea using new HPLC-MS-MS method, J. Pharm. Biomed. Anal. 131 (2016) 140-145.

[6] P. Svoboda, H. Vlčková, L. Nováková, Development and validation of UHPLC-MS/MS method for determination of eight naturally occurring catechin derivatives in various tea samples and the role of matrix effects, J. Pharm. Biomed. Anal. 114 (2015) 62-70.

[7] M. Naldi, J. Fiori, R. Gotti, A. Périat, J.-L. Veuthey, D. Guillarme, V. Andrisano, UHPLC determination of catechins for the quality control of green tea, J. Pharm. Biomed. Anal. 88 (2014) 307-314.

[8] C. Fanali, A. Rocco, Z. Aturki, L. Mondello, S. Fanali, Analysis of polyphenols and methylxantines in tea samples by means of nano-liquid chromatography utilizing capillary columns packed with core-shell particles, J. Chromatogr. A 1234 (2012) 38-44

[9] H. Horie, K. Kohata, Analysis of tea components by high-performance liquid chromatography and high-performance capillary electrophoresis, J. Chromatogr. A 881 (2000) 425-438.

[10] K. Fraser, G.A. Lane, D.E. Otter, Y. Hemar, S.-Y. Quek, S.J. Harrison, S. Rasmussen, Analysis of metabolic markers of tea origin by UHPLC and high resolution mass spectrometry, Food Res. Int. 53 (2013) 827-835.

[11] Y. Zhao, P. Chen, L. Lin, J.M. Harnly, L. Yu, Z. Li, Tentative identification, quantitation, and principal component analysis of green pu-erh green, and white teas using UPLC/DAD/MS, Food Chem. 126 (2011) 1269-1277.

[12] R. Gotti, S. Furlanetto, S. Lanteri, S. Olmo, A. Ragaini, V. Cavrini, Differentiation of green tea samples by chiral CD-MEKC analysis of catechins content, Electrophoresis 30 (2009) 2922-2930.

[13] B. Pasquini, S. Orlandini, M. Goodarzi, C. Caprini, R. Gotti, S. Furlanetto, Chiral cyclodextrin-modified micellar electrokinetic chromatography and chemometric techniques for green tea samples origin discrimination, Talanta 150 (2016) 7-13.

[14] Y. Chen, Y. Jiang, J. Duan, J. Shi, S. Xue, Y. Kakuda, Variation in catechin contents in relation to quality of 'Huang Zhi Xiang' Oolong tea (Camellia sinensis) at various growing altitudes and seasons, Food Chem. 119 (2010) 648-652.

[15] M. Mirasoli, R. Gotti, M. Di Fusco, A. Leoni, C. Colliva, A. Roda, Electronic nose and chiral-capillary electrophoresis in evaluation of the quality changes in commercial green tea leaves during a long-term storage, Talanta 129 (2014) 32-38.

[16] D. Türközü, N. Sanlier, L-Theanine, unique amino acid of tea, and its metabolism, health effects, and safety, Crit. Rev. Food Sci. Nutr. 57 (2017) 1681-1687.

[17] X. Wan, Z. Zhang, D. Li, Chemistry and biological properties of theanine, in: C.-T. Ho, J.-K. Lin, F. Shahidi (Eds.), Tea and Tea Products. Chemistry and Health-Promoting Properties, CRC Press, Boca Raton, FL, 2009, pp. 255-274.

[18] Q.V. Vuong, M.C. Bowyer, P.D. Roach, L-Theanine: properties, synthesis, and isolation from tea, J. Sci. Food Agric. 91 (2011) 1931-1939.

[19] ISO 19563:2017(en). Determination of theanine in tea and instant tea in solid form using high-performance liquid chromatography. ISO/TC 34/SC 8. ICS: 67.140.10.

[20] C.-N. Chen, C.-M. Liang, J.-R. Lai, Y.-J. Tsai, J.-S. Tsay, J.-K. Lin, Capillary electrophoretic determination of theanine caffeine, and catechins in fresh tea leaves and oolong tea and their effects on rat neurosphere adhesion and migration, J. Agric. Food Chem. 51 (2003) 7495-7503.

[21] H. -Yi Hsiao, R.L.C. Chen, T.-J. Cheng, Determination of tea fermentation degree by a rapid micellar electrokinetic chromatography, Food Chem. 120 (2010) 632-636.

[22] J. Yan, Y. Cai, Y. Wang, X. Lin, H. Li, Simultaneous determination of amino acids in tea leaves by micellar electrokinetic chromatography with laser-induced fluorescence detection, Food Chem. 143 (2014) 82-89. 
[23] M.-M. Hsieh, S.-M. Chen, Determination of amino acids in tea leaves and beverages using capillary electrophoresis with light-emitting diode-induced fluorescence detection, Talanta 73 (2007) 326-331.

[24] A. Alcázar, O. Ballestreros, J.M. Jurado, F. Pablos, M.J. Martín, J.L. Vilches, A Navalón, Differentiation of green, white, black, oolong, and pu-erh teas according to their free amino acids content, J. Agric. Food Chem. 55 (2007) $5960-5965$.

[25] L. Wang, R. Xu, B. Hu, W. Li, Y. Sun, Y. Tu, X. Zeng, Analysis of free amino acids in chinese teas and flower of tea plant by high performance liquid chromatography combined with solid-phase extraction, Food Chem. 123 (2010) 1259-1266.

[26] R. Thippeswamy, K.G. Mallikarjun Gouda, D.H. Rao, A. Martin, L.R. Gowda, Determination of theanine in commercial tea by liquid chromatography with fluorescence and diode array ultraviolet detection, J. Agric. Food Chem. 54 (2006) 7014-7019.

[27] R. Horanni, U.H. Engelhardt, Determination of amino acids in white, green, black, oolong pu-erh and tea products, J. Food Comp. Anal. 31 (2013) 94-100.

[28] K.H. Ekborg-Ott, A. Taylor, D.W. Armstrong, Varietal differences in the total and enantiomeric composition of theanine in tea, J. Agric. Food Chem. 45 (1997) 353-363.

[29] R. Horanni, U.H. Engelhardt, Enantiomeric analysis of theanine in different teas (Camellia sinensis) using Marfey’s reagent, Eur. Food Res. Technol. 240 (2015) 61-70.

[30] Y. Zhao, P. Li, L. Ge, Y. Wang, T. Mo, X. Zeng, X. Wang, Effect of electrolyzed reduced water on chiral theanine and polyphenols in tea, Food Chem. 134 (2012) 1761-1766.
[31] M.J. Desai, D.W. Armstrong, Analysis of derivatized and underivatized theanine enantiomers by high-performance liquid chromatography/atmospheric pressure ionization-mass spectrometry, Rapid Commun. Mass Spectrom. 18 (2004) 251-256.

[32] R. Ito, A. Yamamoto, S. Kodama, K. Kato, Y. Yoshimura, A. Matsunaga, H Nakazawa, A study on the change of enantiomeric purity of catechins in green tea infusion, Food Chem. 83 (2003) 563-568.

[33] R. Gotti, S. Furlanetto, S. Pinzauti, V. Cavrini, Analysis of catechins in Theobroma cacao beans by cyclodextrin-modified micellar electrokinetic chromatography, J. Chromatogr. A 1112 (2006) 345-352.

[34] S. Terabe, Micellar electrokinetic chromatography, Anal. Chem. 76 (2004) 240A-246A

[35] S. Terabe, Y. Miyashita, Y. Ishihama, O. Shibata, Cyclodextrin-modified micellar electrokinetic chromatography: separation of hydrophobic and enantiomeric compounds, J.Chromatogr. 636 (1993) 47-55.

[36] D. Kutlán, I. Molnár-Perl, Characteristics and stability of the OPA/3-mercaptopropionic acid and OPA/N-acetyl-L-cysteine derivatives of amino acids, Chromatographia 53 (2001) S188-S198.

[37] Y. Mengerink, D. Kutlán, F. Tóth, A. Csámpai, I. Molnár-Perl, Advances in the evaluation of the stability and characteristics of the amino acid and amine derivatives obtained with the o-phthaldialdehyde/3-mercaptopropionic acid and o-phthaldialdehyde/N-acetyl-L-cysteine reagents. High-performance liquid chromatography-mass spectrometry study, J. Chromatogr. A 949 (2002) 99-124.

[38] ICH Harmonised Tripartite Guideline, Q2(R1), Validation of Analytical Procedures, 2005. 